\title{
Principes d'une grammaire prédictive du discours (français langue étrangère et maternelle)
}

\author{
Muriel Barbazan \\ Laboratoire Jacques-Lordat \\ Université Toulouse-Le Mirail \\ muriel.barbazan@univ-tlse2.fr
}

\begin{abstract}
La grammaire actuelle a du mal à articuler la syntaxe traditionnelle aux investigations plus récentes des « analyses de discours », que développent la linguistique énonciative (interactionniste) et la linguistique textuelle, secondée par la psycholinguistique. Ces difficultés sont encore plus marquées en FLE que dans le domaine du FLM. Afin de comprendre les raisons de ces difficultés intégratives gênantes tant sur le plan théorique que sur le plan didactique, nous explorerons les contraintes héritées du structuralisme de Saussure et les débordements paradigmatiques énonciatifs et textuels que devait inévitablement générer ce cadre trop restreint. Ce parcours préalable mettra en évidence l'origine épistémologique de l'hétérogénéité actuelle des domaines grammaticaux, mais aussi le paradoxe de leur complémentarité indissociable du point de vue cognitif (développement ontologique et gestion mentale du langage). Il sera alors possible de justifier les principes d'une reformulation descriptive articulant l'héritage syntaxique aux fonctions énonciative et de structuration du texte, tant au niveau de la globalité textuelle qu'au niveau des moyens grammaticaux qui en permettent l'élaboration (lexique, connecteurs, système verbal, pronoms / renominalisations, syntaxe phrastique, unités de structure du texte).
\end{abstract}

\section{Introduction}

La grammaire, plus encore en français langue étrangère (FLE) qu'en français langue maternelle (FLM), a du mal à se présenter comme un domaine homogène, qui articule de façon pertinente l'héritage traditionnel et les avancées récentes de la linguistique énonciative et des grammaires de texte. Si le FLM semble être en meilleure voie de réorganisation (Garcia-Debanc et al. 2001), le FLE a des difficultés à intégrer en un système cohérent des notions énonciatives ou textuelles encore trop parcellisées, et qui ne sont pas reliées aux connaissances grammaticales traditionnelles : on est face à une juxtaposition de plusieurs systèmes, sans fil conducteur permettant de repérer dans cet ensemble un cheminement cohérent. Il faudrait pouvoir sortir de cette impression «d'emboîtement de différents dispositifs d'organisation de la langue, du discours à l'énoncé, du texte à la phrase, même si l'on ne sait pas encore très bien comment s'articulent dimension discursive ou énonciative et dimension formelle de l'organisation de la langue » (Vigner 2004, 86). Un des défis actuels posés au grammairien est donc de tenter d'homogénéiser ces divers courants en une discipline plus cohérente. Tant que ce n'est pas fait, l'apprenant n'a pas d'autre choix que d'essayer d'articuler comme il peut ses connaissances entre elles :

«Il appartiendra toujours aux élèves d'établir les liaisons nécessaires entre ces différents niveaux d'organisation de la langue (du discours aux éléments de la phrase ou inversement), l'essentiel étant de proposer des activités qui faciliteront cette mise en relation. » (Vigner 2004, 86)

Ce point de vue nous parait tout de même un peu défaitiste, car il revient à situer la didactique en aval de modélisations qui ne concernent pas la seule linguistique. Deux raisons semblent justifier un point de vue 
plus optimiste quant au devenir et aux ambitions de la grammaire : la première est que la voie a été ouverte par la grammaire de FLM. Ainsi, l'articulation énonciation / texte / phrase fait déjà l'objet d'une problématisation engagée par de nombreux chercheurs linguistes et / ou didacticiens, et ces efforts intégratifs se sont traduits dans les programmes et les manuels de FLM. La seconde raison, c'est que les échanges interdisciplinaires peuvent faciliter le cheminement réflexif en mettant par exemple au service $\mathrm{du}$ didacticien et du linguiste des connaissances solides en psychologie cognitive (traitement du texte ou ici «discours » - en compréhension et production, processus d'apprentissage et activation des connaissances en mémoire etc.). La pluridisciplinarité peut ainsi permettre de mieux contrôler la rigueur de la démarche, et de repérer certains écueils qu'un éclairage unidisciplinaire ne permettrait pas de voir.

Si l'on a cette impression générale de manque de cohérence interne, c'est parce que les modalités de fonctionnement linguistique décrites dans les domaines envisagés relèvent de paradigmes différents. Les linguistiques énonciatives et textuelles se sont construites en s'opposant aux postulats immanentiste et phrastique du structuralisme originel. Pour autant, l'énonciation et le texte ne se sont pas immédiatement reconnus l'un l'autre pour s'intégrer a priori dans un cadre de travail conjoint unifié. L'unification d'un parcours grammatical passe vraisemblablement par l'établissement d'un cadre d'investigation suffisamment large pour intégrer les présupposés énonciatifs et textuels, tout en absorbant les connaissances formulées en grammaire traditionnelle. Parfois, comme on va le voir, un simple changement d'éclairage permet de rétablir certaines proportions sémantiques, exagérément hypertrophiées par le point de vue traditionnel. Dans cette perspective, il est utile de partir d'assez loin : on fera ainsi un rappel de l'héritage immanentiste, descriptif et phrastique pour montrer les forces d'inerties que ce paradigme génère, et qui contribuent à freiner voire à bloquer les tentatives d'intégrer de manière globale les dimensions énonciatives et textuelles dans le champ linguistique. On pourra alors mieux comprendre les raisons du manque d'homogénéité des domaines couverts par la grammaire (phrase / texte / énonciation), pour proposer ensuite des perspectives d'homogénéisation.

\section{L'héritage de l'immanentisme descriptif et phrastique}

L'un des objectifs majeurs du structuralisme hérité de Saussure a été de situer la réflexion linguistique dans l'abstraction d'un cadre axiomatiquement fermé, celui de la langue «en soi et pour soi», en évacuant «l'ensemble hétéroclite des faits de langage ». Et notamment, l'utilisation même du système de la langue, en tant qu'elle implique de prendre en compte des individus parlant, relève des avatars de la parole, et ne constitue pas à ce titre l'objet premier de la linguistique. En d'autres termes, il s'agit d'appréhender la langue en dehors de son usage, ce qui ne peut pas manquer de plonger dans la perplexité quiconque admet que les interactions menées par le biais du langage font cognitivement partie intégrante de ses propriétés, dont elles servent à forger les représentations conceptuelles (Vigotsky 1934, Bruner 1983, Mondada / Pekarek 2000).

Par ailleurs, en évacuant d'emblée la fonction communicative du langage hors du champ d'investigation linguistique, on a implicitement confirmé la représentation déjà bien établie qui veut que la fonction première de la langue soit de référer au monde, par l'intermédiaire du « signe », descriptible dans son aboutissement selon la trilogie signifié / signifiant / référent. L'idéalisme artificiel de cette position a été dénoncé tant au niveau linguistique (Berrendonner 1981, 36ss ; Kleiber 1997) qu'en psychologie, notamment par les psychologues gestaltistes. Avant même de mettre en question l'absence d'ancrage énonciatif présumé pour la fonction référentielle (ou dénotative), nous nous contentons ici de souligner l'hypertrophie artificielle de cette fonction au sein du structuralisme.

Pour compléter la portée réductrice et contraignante de ce paradigme de la «langue » et du « signe », on notera que la combinatoire des signes entre eux, à savoir la morphosyntaxe et la syntaxe ne dépasse pas les limites de la phrase. Le texte et ses composantes structurelles propres ne font pas a priori partie des interrogations linguistiques.

Ces trois contraintes paradigmatiques : l'artificielle opposition langue / parole assortie de l'hypertrophie d'une fonction référentielle illusoirement autonome et de l'étroitesse du cadre phrastique pèsent encore lourdement dans l'inconscient collectif des linguistes et des didacticiens. 
À cet héritage immanentiste, il nous paraît nécessaire de répondre par un engagement fonctionnaliste clairement revendiqué, un fonctionnalisme qui n'oppose pas syntaxe (plus ou moins formelle) et sémantique, et qui ne se focalise pas sur la fonction informative du langage et sa dimension référentielle. Ce point de vue rejoint celui de Charolles et Combettes :

«Dans les dernières décennies, sous la pression de modèles géométriques de la syntaxe, on a sans doute eu tendance à présenter la grammaire sous un angle excessivement structuraliste, en oubliant que les principes qui gouvernent l'agencement des morphèmes au sein de la proposition et de la phrase ont aussi, et probablement, avant tout, une portée fonctionnelle et une origine cognitive. » (Charolles et Combettes 2001, 139, c'est nous qui soulignons)

\section{La quadrature du cercle saussurien : intégrer l'énonciation et le texte}

L'adoption d'un point de vue fonctionnaliste a d'ailleurs été régulièrement revendiquée dès l'émergence du courant énonciatif, et notamment par Benveniste, considéré comme un des initiateurs de ce courant réactif au postulat immanentiste en France :

«L'énonciation est cette mise en fonctionnement de la langue par un acte individuel d'utilisation. » (Benveniste 1974, 80)

Dans la lignée de Benveniste, la parution de l'Énonciation de Kerbrat-Orecchioni $(1980)^{1}$ constitue un jalon de l'avènement du cadre énonciatif. Elle y souligne le caractère fondamentalement fonctionnel du langage, impossible à appréhender en excluant a priori les acteurs du discours, et poursuit l'exploration de l'inscription énonciative au sein même de catégories lexicales et grammaticales.

Pour autant, comme on va le voir, la constitution d'une «analyse du discours », envisagée comme un champ englobant à la fois le domaine énonciatif et le domaine textuel, ne s'est pas imposée en corrélation avec une extension ou une redéfinition paradigmatique explicite. Il est alors utile de mettre en évidence quelques options et paliers intégratifs notoires marquant l'avancée de ces champs d'analyse dans le domaine linguistique pour mieux comprendre l'inertie que constitue encore l'héritage du structuralisme immanentiste et des habitudes conceptuelles plus ou moins conscientes qu'il nourrit. Sous l'éclairage de ces options et tournants constitutifs de la linguistique énonciative et textuelle actuelle, on peut espérer mieux cerner les difficultés auxquelles achoppe la constitution d'une grammaire réellement discursive.

\subsection{Tentatives de sauvetage du legs immanentiste}

Devant l'irrépressible ascension du courant énonciatif, diverses options ont été envisagées pour résoudre l'embarras que pose la transmission de l'héritage structuraliste tout en réglant la succession de la façon la plus économique possible, pour ce qui est de son intégration à un nouveau paradigme.

L'option apparemment la plus simple consiste à proclamer un lien énonciatif fondamental rattachant les productions linguistiques au locuteur / récepteur tout en réintégrant les représentations sémantiques traditionnelles telles quelles ou "relookées" sous une apparence plus séduisante au plan énonciatif. La gestion de l'héritage se fait apparemment à moindre coût et se limite pratiquement à des redénominations terminologiques superficielles : la "phrase » devient «énoncé », le «texte ${ }^{2}$ » devient « discours ». Mais les bénéfices d'une telle manipulation sont à la hauteur de l'investissement théorique. Force est de constater qu'il ne suffit pas de décréter que le locuteur et éventuellement le récepteur sont intégrés au modèle pour qu'ils le soient. Un indice de leur évanescence est bien que rien d'autre n'a concrètement changé sinon le décret de leur présence : le fond de la description linguistique s'articule selon des rouages identiques, et le cadre énonciatif revendiqué s'apparente plus à une proclamation politique qu'à une modification paradigmatique effective. L'intégration d'un locuteur idéalisé dans la compétence chomskienne n'a généré qu'un mince décalage par rapport au concept fondateur de la langue structuraliste. On peut évoquer ici

\footnotetext{
${ }^{1}$ Citée ici dans la troisième édition de 1997.

${ }^{2}$ Ici au sens trivial de fragment linguistique décontextualisé de la situation de production.
} 
aussi le fonctionnalisme "minimaliste" de Martinet, qui certes met l'accent sur la fonction de communication du langage, mais qui retrouve de fait les réductions héritées du passé : d'abord, la communication envisagée est essentiellement informative, au dépend des autres fonctions du langage. Cette restriction a pour effet de conserver un éclairage focalisé sur la dimension référentielle / descriptive du langage - qui réfère à une réalité extra-linguistique pré-existant au message - au dépend de l'analyse des effets de sens effectivement liées à l'activité communicative du producteur et du récepteur. Corrélativement, et de façon cohérente dans cette perspective, le code est assimilé à un instrument servant à la transmission de messages indépendamment des particularités énonciatives des acteurs linguistiques. On récupère de fait la partition langue / parole :

«[...] le message est assimilé à une réalité quasi-matérielle pré-existant à la communication, comme un objet qui passerait de main en main [...]. A ce compte [...], les règles d'organisation des messages selon le code de la langue peuvent être étudiées indépendamment des activités d'encodage et de décodage. » (Fuchs et Le Goffic 1992, 131)

Dans un champ connexe à ces tentatives timidement émancipées des postulats héritées du passé, il existe une perspective d'analyse plus engagée dans la voie d'une sémantique qui se préoccupe d'avantage de l'impact de la situation de production / réception sur la construction du sens des énoncés. Cette voie, encore empruntée aujourd'hui ${ }^{3}$, consiste à faire le pari de la conservation possible d'un signifié de langue, en le considérant comme la signification «première », ou « littérale » de l'élément linguistique concerné. À cette base sémantique fondamentale envisagée hors discours, on ajoute des compléments de signification d'ordre pragmatique (couvrant le domaine énonciatif) à partir des informations particulières à une situation de production donnée. Il faut bien voir que dans cette perspective, on conserve intact le signifié linguistique hérité. Ducrot (1991), dans son avant-propos à la troisième édition de Dire et ne pas dire (1972), rappelle qu'il avait revendiqué ce type de modèle à l'époque de la première parution de cet ouvrage. L'objectif était donc de défendre

«l'idée qu'il y a, dans la construction du sens, un moment strictement linguistique, où l'on attribue une valeur à la phrase, et un second, que j'appelais "rhétorique", où cette première valeur interagit avec la situation. »

Il s'agissait bien d' « imaginer, peut-être même construire une machine composée de deux modules, [...] bien délimités : l'un traitait tout ce qui vient de la langue, c'est-à-dire tout ce qui est inscrit dans la phrase que l'énoncé réalise, l'autre combinait les résultats du premier avec les données du contexte et de la situation, et arrivait à reconstruire le "sens effectif" de l'énoncé, tel que 1'“observation" l'avait révélé. » (Ducrot 1991, Avant-propos)

Pourtant, entre les dates de parution des deux éditions citées et à la suite du développement de ce modèle bi-polaire, Ducrot constate que la distinction entre les deux modules n'est plus aussi évidente :

«À force d'être travaillées, ces distinctions ont perdu l'heureuse netteté d'autrefois : les limites se sont mises à trembler. » (ibid.)

Ces difficultés sont loin d'être anodines, car ce peut être le signe que le fonctionnement effectif du langage résiste à ce principe dualiste qui devait permettre de conserver intact les acquis du passé : la langue comme système de signes, représentée par la composante «strictement linguistique » et l'intégration $a$ posteriori d'un module rhétorique distinct, dépendant de données particulières à chaque situation de production (cf. Ducrot 1991, 111s). Cette représentation sémantique modulaire, dite «théorie en Y » par Berrendonner, se heurte à l'existence d'éléments linguistiques impliquant précisément du sens énonciatif dans leur signification première, littérale (cf. Berrendonner 1981, 13 $3^{4}$ ). Dans ce cas effectivement, l'opposition sens en langue / sens en situation n'a plus lieu d'être. Elle génère notamment le paradoxe de catégories linguistiques comme les indiciels (les pronoms ou les adverbes déictiques par exemple) qui constituent des îlots sémantiques dont le signifié implique un lien à une situation d'actualisation, quelle qu'elle soit - alors qu'on se trouve dans un modèle qui postule précisément des signifiés littéraux hors du

\footnotetext{
${ }^{3}$ Cf. notamment les travaux effectués à la suite de Sperber et Wilson (1989), et de la « Théorie de la pertinence ».

${ }^{4}$ Pour une discussion d'autres exemples de difficultés que rencontre le modèle de Ducrot, voir Berrendonner (1981, 9ss et Kerbrat-Orecchioni 1997, 196ss).
} 
champ de l'actualisation du langage. Dès 1980, Kerbrat-Orecchioni rencontre une incompatibilité intégrative de ses propres résultats dans ce modèle. Elle évoque en l'occurrence la catégorie des lexèmes qui renvoient dans leur signification même à une position évaluative négative de l'énonciateur par rapport au contenu référentiel.

«Faut-il en conclure que le trait de dévalorisation dont ils sont porteurs relève de la composante pragmatique ? Mais ce trait évaluatif est proprement indissociable des autres ingrédients descriptifs du sémème. Doiton admettre la possibilité, pour une même unité de contenu, de jouer à la fois sur les deux tableaux sémantique et pragmatique ?»(Kerbrat-Orecchioni 1997, 199)

Cette dernière question, qui nous parait aujourd'hui centrale, sera réenvisagée plus loin. On peut faire pour l'instant un bilan intermédiaire concernant l'origine de l'hétérogénéité des domaines grammaticaux. La syntaxe traditionnelle est profondément marquée par le postulat immanentiste, dans lequel on ne peut pas intégrer certains «îlots » énonciatifs qui résistent dans leur signification et leur fonctionnement à la dissociation langue / usage de la langue. De plus, ce qui a paru d'abord pouvoir être considéré comme des îlots somme toute restreints (les verbes performatifs et les actes « déclaratifs » au sens de Searle, les déictiques, les registres de langage familiers ou standard...) s'est transformé progressivement en continent énonciatif et textuel. Si l'héritage du passé doit être capitalisé, ce n'est donc vraisemblablement pas dans un cadre dissociant fondamentalement la langue comme système en soi de son utilisation par les usagers.

Dans ce parcours exploratoire de l'articulation des fonctions référentielle et énonciative du langage, il est intéressant de souligner un effet de "balancier épistémologique" qui conduit Benveniste à nier le contenu référentiel des pronoms personnels, envisagés dans leur seule fonction de supports énonciatifs nécessairement actualisés. Kerbrat-Orecchioni relève ainsi cette étonnante et célèbre affirmation :

« Les pronoms [...] ne renvoient ni à un concept ni à un individu. »(Benveniste 1966, 261)

Comme le fait remarquer Kerbrat-Orecchioni (1997, 44), « on peut contester l'expression de “formes vides" qu'utilise Benveniste au sujet des pronoms ». Ils ont effectivement bien un sens conceptuel : je par exemple renvoie au producteur de l'énoncé dans lequel apparaît son occurrence. Peu importe si le référent n'est pas fixe ni intrinsèquement identifiable hors du discours. La rectification est effectivement nécessaire, et si cette affirmation paraît surprenante de la part de Benveniste, elle peut s'expliquer en réaction à l'idéal référentiel objectiviste, qui postule un référent stable hors du discours contre lequel s'insurge ainsi Benveniste. Comme on le voit, cette définition exclusivement énonciative des pronoms n'est pas seulement anecdotique, et elle tout nous paraît symptomatique d'une difficulté toujours actuelle : comment envisager le signifié des éléments linguistiques ? Comment articuler le langage comme trace de la relation énonciateur / récepteur et le langage comme outil de référence au monde (réel ou imaginaire) ? Si le processus énonciatif laisse des traces dans le produit de l'énonciation, il faut pourtant que les éléments linguistiques qui servent de support à ces effets de sens intègrent dans leurs signifiés un trait sémantique responsable de ces effets.

Pour conclure ce parcours rapide d'un problème hérité de longue date, il apparaît qu'il est encore courant de privilégier une distinction exclusive entre ces deux pôles. Soit on met l'accent sur la fonction référentielle (renvoyant au monde extra-linguistique) en retrouvant la trilogie signifié / signifiant / référent développée d'ailleurs de façon très fine jusque dans la théorie des prototypes (Dubois 1993), soit on se préoccupe de la fonction énonciative de certains outils linguistiques, sans nécessairement articuler au sein même des signifiés ce sens énonciatif à leur sens référentiel. La solution théorique initiée par Ducrot ne fait que souligner ce hiatus essentiel, sans le résoudre. Peut-être faudrait-il reconnaître que l'on s'enferre dans une dichotomie artificielle entre deux fonctions sémantiques complémentaires et irréductibles l'une à l'autre. Ce sont ces deux fonctions qu'il faut arriver à réconcilier pour homogénéiser enfin le champ grammatical, abandonné dans l'éther de l'immanentisme et le champ énonciatif, largement développé par les linguistes et didacticiens interactionnistes, mais sur des chemins le plus souvent assez éloignés d'une franche volonté de rassembler sous un même paradigme l'ensemble des domaines grammaticaux (cf. Véronique / Vion 1995, Arditty / Vasseur 1999, et bien sûr la revue AILE). C'est à ce prix que l'on pourra espérer répondre à cette question parallèle qui nous préoccupe sur le versant grammatical en didac- 
tique : comment homogénéiser les représentations décontextualisées de la grammaire et les connaissances issues des recherches effectuées en linguistique énonciative et textuelle?

\subsection{Maturation progressive de deux frères siamois : l'énonciation et le texte}

La première époque de la linguistique énonciative a permis de faire enfin vaciller le cadre immanentiste traditionnel en osant remettre en question la distinction langue / parole (ou compétence / performance) par une revendication de la «mise en fonctionnement de la langue par un acte individuel d'utilisation » (Benveniste 1974, 80). Si une voie énonciative s'est ainsi ouverte, on est encore loin du dialogisme revendiqué par F. Jacques (1979, 1985), qui milite pour la prise en compte corrélative de l'énonciateur et du récepteur. Mais l'impulsion essentielle est donnée et on passe progressivement d'une conception monologique de l'énonciation à une conception effectivement dialogique, qui met en évidence dans les textes des traces de prise de position de l'énonciateur par rapport au contenu de son discours, mais aussi en fonction du récepteur. Celui-ci joue un rôle de toute première importance dans la constitution même du discours, tant sur la forme que sur le fond, à l'oral aussi bien qu'à l'écrit. Les travaux initiés par Kerbrat-Orecchioni sur les interactions verbales ont été depuis lors largement suivis et suffisemment développés pour qu'on n'y revienne pas ici en détail.

À ce stade de la réflexion, il est temps de se tourner vers deux questions qui prolongent logiquement celle de l'articulation de la langue et du champ énonciatif: comment envisager la transition de la phrase au texte ? Comment articuler aussi les domaines énonciatif et textuel ?

Ainsi que le montrent Charolles et Combettes dans leur article déjà cité $(2001,124)$, le passage de la phrase au texte ne s'est pas fait sans heurt, et l'on ne peut d'ailleurs pas considérer qu'il soit abouti aujourd'hui. En effet, l'avènement du texte s'est d'abord affirmé à travers une opposition stricte vis-à-vis de la grammaire phrastique : le texte «n'est pas une unité grammaticale, mais une unité "d'usage du langage" » (Halliday et Hasan 1976, 1 cités par Charolles et Combettes 2001, 122).

Pourtant, ici encore, de nombreux aspects cognitifs justifient une prise en compte du texte comme unité de fonctionnement à part entière, tant au niveau linguistique qu'au niveau didactique, et non comme simple juxtaposition de phrases. Sur la question des caractéristiques structurales du texte, nous renvoyons aux travaux pionniers de Mandler / Johnson, van Dijk, Kintsch rassemblés dans Denhière (1984), à la suite desquels il faut citer aussi les diverses publications de Adam (notamment 1992, 1999), Fayol (1985, 1997), Fayol et al. (1992), suffisamment connues aussi bien par les linguistes que par les didacticiens et les psychologues pour qu'elles puissent constituer un fond commun et ancrer une discussion pluridisciplinaire solide. En deux mots, la linguistique textuelle s'est attachée à définir types et genres de textes, mais aussi à comprendre les particularités structurales d'unités constitutives des plans de textes, ainsi que leur articulation - c'est-à-dire la relation entre cohérence sémantique et cohésion textuelle.

Parallèlement à l'émergence progressive d'une grammaire textuelle, au départ peu soucieuse d'intégrer l'héritage phrastique et les préoccupations descriptives de la grammaire traditionnelle, force est de constater que l'ouverture énonciative, quant à elle, ne s'est pas préoccupée d'intégrer le texte dans la définition de son champ d'investigation. Au contraire, l'énonciation se définit à ses débuts en niant l'existence d'unités textuelles supérieures à la proposition :

«La phrase [pour] Benveniste (1962/74), ne peut “entrer à titre de partie dans une totalité de rang plus élevé. Une proposition peut seulement précéder ou suivre une autre proposition dans un rapport de consécution" (i.e. de successivité) car "un groupe de propositions", autrement dit un discours, "ne constitue pas une unité d'un ordre supérieur à la proposition" (p.129). » Charolles et Combettes $(2001,121)$

Si Benveniste n'exclut pas l'analyse de larges fragments linguistiques, c'est seulement pour mettre en évidence « comment le sujet s'approprie l'appareil formel de la langue et énonce sa position de locuteur par des indices spécifiques » (Benveniste, cité par Charolles et Combettes 2001, 121). La distinction des registres énonciatifs histoire et discours illustre les manifestations énonciatives que l'on peut mettre en évidence dans cette perspective, manifestations dont la généralité tient effectivement au fait qu'elles ne 
sont pas associées à un plan de texte particulier ${ }^{5}$. Cette dissociation entre perspective énonciative et perspective textuelle, qui paraît aujourd'hui un peu excessive, n'est certes plus d'actualité. Il est permis de penser qu'elle était une étape nécessaire à la constitution de deux domaines distincts, dont on a pu seulement ensuite mettre en évidence la complémentarité. Pour autant, la question cruciale pour le grammairien reste celle de l'articulation de l'héritage syntaxique à ces deux domaines, qui se développent par ailleurs actuellement dans une relation de fraternité plus sereine, plus ou moins explicitement hors du cadre saussurien.

Notre examen nécessairement rapide de l'héritage linguistique, de la grammaire phrastique et décontextualisée aux champs énonciatif et textuel, a permis de faire des hypothèses sur les raisons épistémologiques qui ont conduit à envisager de façon non intégrative ces trois domaines. Or, il apparaît que leur articulation dans un ensemble cohérent est nécessaire pour envisager les grandes lignes d'une grammaire discursive effectivement fonctionnelle. Mais avant d'aborder ce qui sera notre dernière partie, il est intéressant de rappeler brièvement quels regards portent didacticiens et psychologues cognitifs sur ces difficultés articulatoires héritées de la linguistique. Nous ne ferons évidemment pas ici un historique de l'évolution des méthodologies didactiques concernant la grammaire, que ce soit pour le FLE ou le FLM. Notre objectif est ici de cadrer les propositions faites en dernière partie en soulignant certaines difficultés essentielles rencontrées dans le domaine de l'apprentissage / enseignement de la grammaire, mais aussi en établissant des repères cognitifs permettant de baliser le cheminement réflexif.

\title{
4 Points de vue de didacticiens et de psychologues
}

Nous avons déjà évoqué en introduction la perplexité que génère chez les grammairiens de FLE la tripartition hétérogène du domaine grammatical, perçue comme un éclatement difficilement conciliable entre les pôles syntaxique, énonciatif et textuel. Vigner $(2004,85)$ formule ainsi une difficulté théorique que Besse et Porquier relevaient déjà vingt ans auparavant :

\begin{abstract}
«Cette extension du champ grammatical à l'ensemble des manifestations de la prise de parole, à l'échange conversationnel ou aux différentes formes d'écriture, peut inquiéter toute personne soucieuse de rigueur dans la description des faits de langue. De nombreuses descriptions, dans tout ce qui se situe dans l'au-delà de la phrase, ou qui sont en relation avec le sujet énonciateur, restent parfois trop ponctuelles ou trop spécifiques à un domaine d'usage pour pouvoir être généralisées à d'autres. Aussi serait-on tenté de partager le point de vue d'H. Besse qui notait que : « ces descriptions, nouvelles mais encore très parcellisées, sont beaucoup plus présentes dans les discours des linguistes et des théoriciens de l'enseignement / apprentissage des langues que dans les manuels et les pratiques de classe, probablement parce qu'elles ont très peu rénové les connaissances morphologiques traditionnelles ou structurales [...]» Besse 1984, 20.» (Vigner 2004, 85).
\end{abstract}

Nous avons exploré précédemment les raisons qui ont fait que les connaissances morphologiques (et morpho-syntaxiques) traditionnelles ou structurales ont été peu rénovées par les apports énonciatif et textuel. On verra aussi plus loin que les contributions de ces deux derniers domaines sont aujourd'hui loin d'être parcellaires, mais empiètent de plus en plus sur le domaine prétendument décontextualisable de la grammaire, comme la définition lexicale ou le fonctionnement de la phrase même.

Les didacticiens du FLM ont abordé de façon plus frontale cette problématique que ne l'ont fait les spécialistes de FLE. C'est donc dans le champ de la didactique du FLM que l'on rencontre les avancées les plus nettes (cf. Vigner 2004, 86 et surtout Garcia-Debanc et al. 2001). Maingueneau $(2001,16)$ rappelle que l'enseignement actuel en FLM « associe traditionnellement une analyse de la langue hors contexte, qui se tient en général dans les limites de la phrase et que l'on a pris l'habitude de nommer "grammaire" [...] et que l'on met en contraste avec des activités centrées sur l'étude et la production de textes. » Dans cette dernière perspective, qui donne «tout son poids à la notion de discours ", le langage est conçu " comme activité, comme interactivité », tant au niveau du champ énonciatif qu'au niveau de la constitu-

\footnotetext{
${ }^{5}$ On sait par ailleurs que la tentative de Weinrich (1982) d'associer les registres récit / discours (hérités de Benveniste) à des types de textes pour en expliciter les contextes d'emploi échoue tant au niveau théorique qu'au niveau didactique (Barbazan 2006, 160ss et 2007b).
} 
tion structurale des textes. Il ajoute que « la difficulté pour les didacticiens est de gérer cette double polarité ». Comment en effet articuler les activités grammaticales et les activités liées aux textes, au « discours » ? D'autre part, dans le sens d'une nécessaire mise en question de l'hypertrophique fonction référentielle (dénoncée plus haut au point 2.), Maingueneau constate :

« le sort exceptionnel qui est réservé [au « maître-mot » discours] tient aussi au fait qu'il permet de contester les conceptions "représentationnistes" du langage jusqu'alors dominantes en linguistique comme en didactique des langues. »

Il n'est guère étonnant de retrouver sur le versant didactique des questions similaires à celles qui préoccupent les linguistes. En deux mots, comment intégrer dans un cadre commun l'héritage structuraliste et les "échappées paradigmatiques" énonciatives et textuelles ${ }^{6}$ ?

En FLE, la question de la sélection et de la présentation des contenus grammaticaux dépend davantage de postulats méthodologiques d'enseignement / apprentissage (cadre audio-visuel, programme notionnel / fonctionnel et méthodes communicatives, cf. Puren 1988, Germain / Netten 1995) que du résultat d'une réflexion particulière sur le fonctionnement du langage et sur le statut de la grammaire par rapport aux « évolutions » des descriptions linguistiques (Puren 1988, 8) ou aux « courants » énonciatifs et pragmatiques (Cuq 1996, 125). Mais si l'on a précisément pour objectif de ne pas noyer les apprenants par un métalangage abscons ou des descriptions grammaticales contre-intuitives ou même fausses, options notoirement contre-productives en FLE, il parait utile de se poser la question de la formulation des contenus grammaticaux en amont de celle de leur didactisation telle qu'elle est actuellement envisagée. Une remarque de Besse et Porquier permet d'illustrer une de nos conclusions personnelles, tant au niveau de la description grammaticale que de l'enseignement du FLE : celle de se donner comme postulat de travail d'établir un lien nécessaire entre la situation d'énonciation et le fonctionnement grammatical du langage :

« Le grammairien classique ne tient pas compte du contexte discursif dont l'énoncé est extrait, ni plus largement de la situation de communication pour laquelle il a été conçu. [...] Et bien qu'adolescents et adultes soient, en principe, capables de saisir ce fonctionnement, nombre d'entre eux résistent, souvent non consciemment, à cette dissociation imposée par l'activité réflexive grammaticale traditionnelle entre l'énonciateur, ses conditions d'énonciation et de réception, et l'énoncé lui-même, simplement parce que cette dissociation n'est pas habituelle, "naturelle", dans l'usage ordinaire des langues [...]. » (Besse / Porquier 1984, 19)

L'articulation essentielle de la grammaire et des pôles énonciatifs / textuels est d'autant plus cruciale en didactique que les apprenants ne perçoivent pas intuitivement "l'existence" d'une langue décontextualisée - si tant est que l'on arrive à démontrer en linguistique le bien-fondé de ce postulat, qui ne relève pas de l'évidence première. Pourtant, depuis la parution de l'ouvrage de Besse / Porquier, il ne semble pas que l'on puisse observer de modification notoire concernant la décontextualisation grammaticale (cf. Vigner 2004, 82).

Les approches communicatives n'échappent pas à cette dichotomie épistémologiquement explicable mais peu fondée cognitivement. Gaonac'h remarque dans ce sens que

« le "communicatif" présente ainsi fréquemment une connotation oppositionnelle (sinon contradictoire) avec le linguistique. Ceci est particulièrement crucial lorsqu'on traite de la "compétence de communication", qui peut apparaître comme une compétence sans lien avec une compétence linguistique (c'est-à-dire portant sur le code linguistique). Cette dichotomie pose problème au psychologue, tout d'abord sur un plan théorique très général. Si langage et communication sont ontologiquement liés, d'un point de vue fonctionnel, on ne voit pas ce qui peut justifier de les étudier séparément, de les mesurer séparément (tests de compétence de communication), de les enseigner séparément (exercices communicatifs). »(Gaonac'h 1991, 177)

\footnotetext{
${ }^{6}$ Nous ne prétendons nullement que tous les problèmes linguistiques sont des problèmes didactiques, et que les questions didactiques se confondent avec celles que se posent les linguistes. Mais pour ce problème précis qui concerne aussi bien le grammairien (de FLE et de FLM) que les concepteurs de manuels, il y aurait tout de même quelque intérêt à ne pas fermer la porte à une collaboration entre linguistique et didactique, au nom de l'ingérence applicationniste passée de la linguistique, fort regrettable, et du légitime objectif des didacticiens à élaborer une discipline à part entière, développant ses propres théorisations.
} 
Vigner regrette l'hétérogénéité existant en linguistique et l'absence de « grande théorie dominante en langue qui permettrait, d'un coup de baguette magique, d'organiser en une totalité cohérente tout le paysage linguistique à parcourir. » (Vigner 2004, 157). En l'absence d'une fée, ou plus prosaïquement d'un paradigme de travail établi, pour " organiser la cohérence » et élaborer une description fonctionnelle et prédictive en FLE, il nous semble judicieux de naviguer au plus près de ce que l'on sait des processus de traitement cognitif du langage, et particulièrement ici des processus de production de texte.

Pour la production monologique (orale ou écrite), que l'on peut considérer comme étant cognitivement plus complexe à gérer que l'échange dialogué, Fayol $(1997,84)$ met en évidence deux domaines de gestion des informations en mémoire de travail. Il s'agit pour le producteur de gérer en parallèle d'une part la linéarité de l'émission linguistique et l'élaboration d'un plan de texte, en relation avec le contenu référentiel; d'autre part, «la situation de production elle-même (les contraintes liées à la situation d'énonciation) et la prise en compte du destinataire ». On retrouve là, au cœur des processus de traitement du langage, ces trois axes qui doivent selon nous être impérativement intégrés et articulés si l'on vise une grammaire adaptée à la production, notamment en FLE. Ces processus reposent sur l'acquisition de routines, dont on peut mettre en évidence l'installation progressive pendant l'enfance et l'adolescence (Fayol 2001, 306ss), une acquisition que l'on souhaite évidemment favoriser par l'enseignement. On peut définir ces routines de traitement mental en termes de stratégies de repérages d'indices congruents appartenant à des catégories variées (de dimensions et de natures diverses: outils grammaticaux, lexique). L'acquisition de ces routines consiste donc à mettre en place dans la mémoire à long terme des schémas correspondant à des "blocs de connaissance" concernant le fonctionnement du langage (cf. Fayol et al. 1992, 74ss). Pour une meilleure efficacité didactique, la description linguistique doit s'efforcer de correspondre au mieux à ce que l'on sait des processus de traitement mental des discours, quitte à remettre en question des habitudes descriptives balisées par des grilles catégorielles réductrices ${ }^{7}$.

\section{Principes d'une grammaire discursive}

Sur la base de ces postulats, on peut résumer en un schéma les trois dimensions complémentaires à prendre en compte. $\mathrm{Ce}$ schéma général ne surprendra personne en ce qui concerne la gestion du texte, envisagé en tant que «discours ». On y retrouve en effet les trois pôles progressivement mis à jour par l'exploration linguistique du fonctionnement du langage.

- La dimension référentielle renvoie au contenu dénoté, son sens, dit-on souvent de façon réductrice, puisque les deux autres dimensions sont autant partie prenante de la signification que le contenu "informatif" (au sens large).

- La dimension énonciative renvoie à la relation énonciateur / récepteur et aux multiples modalités évaluatives du contenu discursif par le producteur. Les indices qui permettent de la déceler dénotent aussi bien un choix conscient qu'une

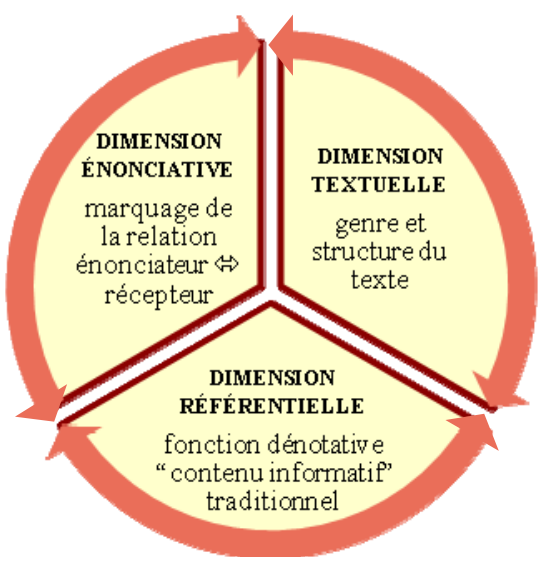

\footnotetext{
${ }^{7}$ Nous pensons par exemple qu'il est nécessaire de sortir du cadre strict de la polyphonie (Maingueneau 1993, Rosier 1999) pour aborder la question du repérage du discours indirect libre (DIL) de façon plus efficace pour les étudiants des concours d'enseignement de langue (français, mais aussi allemand, notamment). Le DIL est en effet très régulièrement "annoncé" en contexte par des indices qui ne relèvent pas du domaine polyphonique, mais qui fonctionnent comme des amorces activant un schéma correspondant à l'émergence textuelle de ce type de discours. Le repérage conscient de ces indices permet de faciliter ensuite au fil du texte l'intégration dans le schéma des marqueurs polyphoniques, dont la seule prise en compte ne suffit souvent pas à désambiguïser la question de l'origine énonciative et donc celle du décodage du DIL, pourtant intuitivement perceptible par les lecteurs experts (cf. Barbazan 2002, à paraître a).
} 
marque énonciative involontaire.

- La dimension textuelle couvre les domaines des genres et types de textes, ainsi que la question de leurs plans organisationnels, et de leurs unités constitutives (séquences...).

Il nous semble nécessaire de souligner deux caractéristiques essentielles de l'articulation de ces dimensions. D'une part, aucune des trois n'est plus importante que les autres : comme on l'a montré plus haut, la surpondération accordée traditionnellement à la fonction référentielle était artificielle. D'autre part, ces trois dimensions sont indissociables, au sens où elles ne peuvent pas entrer en contradiction entre elles dans un texte cohérent et en dehors d'un jeu langagier. Ce lien de cohérence est symbolisé dans le schéma par les doubles flèches, liant chaque dimension aux deux autres. Pour la compréhension des textes, comme pour la prédiction en FLE, on peut alors s'appuyer sur cette cohérence multi-dimensionnelle et développer des stratégies de repérage d'indices congruents relevant simultanément de ces trois dimensions. Ces faisceaux d'indices peuvent permettre de faciliter et de justifier des choix interprétatifs ou productifs. Ainsi que le souligne Gaonac'h :

«Les stratégies de repérage d'indices peuvent être considérées comme des stratégies de communication, dans la mesure où elles consistent à élaborer des hypothèses qui sont supportées en fait essentiellement par des connaissances préalables (de contenu ou formelles) supposées partagées par l'auteur et le lecteur. » (Gaonac'h 1991, 177)

Dans ce contexte épistémologique, il semble alors justifié de militer pour une grammaire textuelle, dont il faut définir bien sûr les modalités en fonction du niveau des apprenants, mais dont le principe paraît acquis. La compréhension du fonctionnement du langage passe par une acquisition du fonctionnement du texte :

« Produire un discours est, d'une certaine façon, plus facile que de produire une phrase, les contraintes discursives, si elles sont maîtrisées suffisamment, pouvant constituer une aide dans la construction des phrases. » (Gaonac'h 1991, 186)

On se trouve donc là en opposition totale avec la grammaire traditionnelle, qui s'ouvre généralement par l'étude des groupes syntaxiques $(\mathrm{GN}, \mathrm{GV} \ldots)$ et se limite souvent à la phrase.

\subsection{Pour un signifié tri-dimensionnel de tous les niveaux fonctionnels du lan- gage}

Au terme de cet article, nous entrons ici dans la partie qui paraîtra probablement la plus surprenante, dans la mesure où elle remet en question la formulation bien établie des significations lexicales et grammaticales. La plupart du temps de façon explicite, on admet pour les outils grammaticaux des signifiés renvoyant avant tout à un contenu dénotatif (référentiel). Ainsi, la fonction des pronoms est de renvoyer au référent installé dans le modèle mental par leur antécédent, les temps verbaux renvoient avant tout à du temps "physique" (fonctions temporelles et / ou aspectuelles), les adverbes et les conjonctions se définissent d'abord par leur contenu sémantique. Quant à la phrase, elle est bien formée si elle est référentiellement cohérente, et elle est généralement envisagée selon un modèle de construction syntaxique standardisé et présenté comme "neutre" par rapport à d'autres constructions "énonciativement marquées" (comme si toute production linguistique n'était pas marquée par la situation d'énonciation, même celles qui se veulent les plus standardisées). Concernant le lexique, sa fonction première est évidemment référentielle aussi, et s'il est marqué énonciativement, on restreint cette fonction aux registres de langage (populaire, familier, standard et soutenu / littéraire). Parallèlement, si l'on veut signaler certaines fonctions énonciatives ou textuelles de ces domaines linguistiques, on le fait dans un second temps et surtout sans relier ces fonctions sémantiques secondaires au signifié des éléments linguistiques déclencheurs de ces effets de sens. À charge pour l'apprenant de tenter une homogénéisation de l'ensemble (Vigner 2004, 86). C'est ainsi que se constitue une catégorie énonciative hétérogène : les registres histoire / discours des temps verbaux, les registres de langage (familier, soutenu...), les modalités, le repérage de la polyphonie, comme le discours indirect libre par exemple, les actes de langage... Au niveau textuel règne la même hétérogénéité. 
«Comment en effet faire comprendre aux élèves que, lorsqu'il s'agit de l'analyse des textes, il peut être utile de regrouper sous la même étiquette de connecteurs un vaste ensemble d'expressions que la grammaire de phrase range dans des catégories différentes (conjonctions, adverbes, groupes prépositionnels, interjections) ? » (Charolles / Combettes 2001, 119)

De la même façon, le traitement textuel de l'anaphore regroupe diverses catégories que « la taxinomie grammaticale distingue soigneusement » (ibid.). (Charolles / Combettes 2001, 119).

Nous avons vu plus haut qu'une entrée discursive dans la grammaire était justifiée cognitivement. On voit ici qu'elle est fondée aussi dans la perspective d'une présentation homogène des catégories d'expressions linguistiques. L'homogénéisation des trois fonctions essentielles à articuler dans l'usage du langage peut être efficacement complétée par l'exploitation du schéma sémantique proposé plus haut à tous les niveaux fonctionnels. On fait là l'hypothèse que ces fonctions cognitives fondamentales dans la gestion du langage s'articulent pour organiser les signifiés des expressions linguistiques au niveau syntaxique (temps verbaux, connecteurs, constructions phrastiques...), mais aussi plus largement au niveau lexical. Dans le cadre restreint de cet article, nous nous limiterons à illustrer de façon sommaire l'intérêt descriptif de cette intégration sémantique.

\subsubsection{Les formes verbales}

Notre parcours exploratoire du fonctionnement des temps verbaux (Barbazan 2002, 2004, 2006...) nous a conduite à envisager un signifié pour les formes verbales similaire à celui que nous avons proposé plus haut pour le texte. Cette représentation sémantique est inspirée de la proposition de Le Ny (1979), qui nous séduit autant par sa rigueur que par sa souplesse de fonctionnement puisqu'elle permet de rendre compte de l'émergence des variations sémantiques contextuelles tout en définissant un invariant conceptuel (cf. Barbazan 2006, 133ss). Le choix de cette représentation sémantique a bien sûr été motivé par une exploration de l'éventail des valeurs contextuelles très diverses prises par les temps du passé, notamment. Cette exploration était d'une part guidée par notre activité d'enseignement du FLE, en Allemagne comme en France, et d'autre part par le refus de tout a priori théorique concernant le sens fondamental des temps (notamment l'adoption onomasiologique d'un paradigme temporel, aspectuel ou au contraire énonciatif, cf. Weinrich 1973). La conclusion générale de cette étude préalable et nécessaire à l'adaptation grammaticale a été que les temps verbaux distribuent leurs valeurs sémantiques selon trois axes : fonctions référentielle, énonciative et textuelle, irréductibles les unes aux autres. En d'autres termes, si les temps verbaux servent effectivement à référer à du temps (chronologique ou aspectuel), ainsi que le prévoit de façon hypertrophiée la perspective traditionnelle, ils sont aussi porteurs d'indices concernant la situation énonciative (Barbazan 2002, 2006, 2007b) et servent à démarquer des parties de texte. Dans cette perspective par exemple, le choix exclusif entre le passé composé (ou passé simple) d'une part et l'imparfait d'autre part peut s'expliquer par une situation en début de 'paragraphe' (séquence ou épisode) pour les deux premiers, qu'il faut opposer à la fonction de cohésion intra-séquentielle pour l'imparfait (Barbazan 2006, 343ss). Une description traditionnelle focalisée exclusivement sur le temps ou l'aspect ne permet pas de rendre compte des contextes énonciatif et textuel, surtout dans une perspective de didactique du FLE (Barbazan 2007a, à paraître b et c).

\subsubsection{Fonctions référentielle, énonciative et textuelle des connecteurs}

Nous entendons par connecteur une classe couvrant diverses catégories hétérogènes de la grammaire traditionnelle (adverbes, conjonctions, groupes prépositionnels, interjections...). Que les connecteurs jouent un rôle essentiel dans la gestion de la structure textuelle ne fait plus de doute aujourd'hui, rôle évidemment en relation avec leur contenu référentiel. En corrélation avec cette fonction textuelle, ils ont aussi un rôle énonciatif. Certains indices, comme le changement de place syntaxique, ou l'environnement sémantique permettent d'activer certains traits du signifié, relevant de telle ou telle dimension de sens (cf. Le Ny 1979, 181ss) : ainsi, l'adverbe bien peut être "adverbe de manière", c'est-à-dire activer au premier plan son sens référentiel (J'ai bien dormi). Il peut devenir "modalisateur" énonciatif ( $\dot{A}$ force de $s$ 'acharner, il a bien fini par avoir son bac $\rightarrow$ effectivement), avec dans ce cas une variation de contenu 
dénotatif. Il peut aussi, en début de partie de texte, marquer une charnière de la structure textuelle (Bien, après cette partie préliminaire, passons au vif du sujet).

Une conjonction comme et, envisagée généralement dans une perspective phrastique, joue aussi un rôle textuel, par exemple comme signal de reprise vocale du narrateur après un fragment au discours indirect libre (Bally, repris par Vuillaume 2000, Barbazan 2008 à paraître a). On peut aussi lui trouver des emplois pour lesquels la dimension énonciative est mise en avant : utilisé dans un texte de façon particulièrement répétitive, il peut être un indice d'une production d'enfant.

Faute de place, nous ne développons pas la multi-fonctionnalité des pronoms, ni de la syntaxe phrastique. On trouvera cependant dans Confais (2001) une illustration convaincante du rôle textuel de la pronominalisation comme marquage cohésif, à l'intérieur d'une unité textuelle, opposée à la renominalisation (le réemploi du nom) située aux charnières structurales dont elle est un indice. Ce rôle justifie que les pronoms soient inclus dans la classe des connecteurs. Parallèlement, de nombreux emplois sont motivés par un choix énonciatif (le choix de nous pour je dans cet article par exemple). Quant à la phrase, l'analyse de sa structure syntaxique peut être interprétée tant au niveau énonciatif que textuel. Dans le premier domaine s'intègrent tous les phénomènes de segmentation, ou de topicalisation, notamment. L'analyse énonciative de la syntaxe phrastique peut être mise en corrélation avec le genre de texte ou le type séquentiel dans lequel elle se situe. Et dans le domaine textuel, on trouve aussi les phénomènes débordant le cadre phrastique, comme les constructions détachées décrites par exemple par Charolles et Combettes, dans leur article déjà cité $(2001,129)$.

\subsubsection{Le lexique.}

La grammaire traditionnelle adopte une attitude ambiguë au regard du lexique. D'une part, on considère qu'il est connexe au domaine qu'elle couvre, et ne concerne donc pas au premier chef le grammairien, mais d'autre part, l'étude des registres de langage (familier, littéraire...), qui passe par le lexique, est généralement envisagée dans les grammaires actuelles. Si l'on veut cependant favoriser l'acquisition de routines de compréhension et de production de textes - en incitant les apprenants à mettre en corrélation sémantique des faisceaux d'indices congruents - il est alors nécessaire de relier l'emploi du lexique à celui des "outils grammaticaux" traditionnels, en relation aussi au domaine textuel (cf. Barbazan 2007b pour une application dans un objectif de didactique en FLE à l'emploi de temps verbaux).

Par ailleurs (ainsi que nous l'avons suggéré au point 3.1.), les conclusions de l'étude de KerbratOrecchioni (1997) vont dans le sens de l'inscription effective d'une dimension énonciative au sein du signifié d'une catégorie lexicale, marquée par un trait [+subjectif], la catégorie des subjectivèmes. Cette dimension énonciative est conjointe à la dimension dénotative (référentielle).

\footnotetext{
«Ces substantifs cumulent deux types d'informations, d'ailleurs indissociables : - une description du dénoté

- un jugement évaluatif, d'appréciation ou de dépréciation, porté sur ce dénoté par le sujet d'énonciation. » (Kerbrat-Orecchioni 1997, 73)
}

Entre autres qualités, que nous ne pouvons pas reprendre ici, ces termes

« sont à éliminer d'un discours à prétention d'objectivité, dans lequel le locuteur refuse de prendre position par rapport au dénoté évoqué. [C'est pourquoi ils] peuvent être considérés comme comportant un trait sémantique [+subjectif] »(Kerbrat-Orecchioni 1997, 73).

Logiquement, et en corrélation avec l'adoption de ce trait [+subjectif] pour certains termes (toutes catégories lexicales confondues), on peut prévoir un trait [-subjectif] pour d'autres. Ces derniers, souvent décrits comme "neutres", alors qu'ils sont aussi dénotatifs d'une attitude énonciative que les subjectivèmes, sont privilégiés par exemple dans les rapports de police. On voit par cet exemple se profiler la possibilité de mettre en relation la "couleur énonciative" d'un terme lexical avec un mode de textualisation privilégié, en relation avec la caractérisation des genres. Il faut bien sûr se méfier ici de la caricature descriptive, péchant par excès de systématisation et source de surgénéralisations inévitables pour les apprenants. 
Dans cette perspective, les traits correspondants aux registres de langue (bagnole vs voiture) sont à inscrire dans la dimension énonciative, de même que peut l'être toute trace d'une appartenance de l'énonciateur à une classe idéologique marquée dans le lexique, ou la trace d'une représentation du récepteur pour le locuteur et de leur relation particulière, associée à un "choix de vocabulaire".

\section{Conclusion}

Au terme de ce parcours rapide, privilégiant un cheminement épistémologique pour souligner le paradoxe cognitif que représentent les difficultés d'intégration du champ traditionnel de la grammaire aux "débordements paradigmatiques" de la linguistique énonciative et textuelle, il nous paraît cohérent de souhaiter fortement l'émergence d'une grammaire discursive, ancrée dans le texte et l'énonciation. L'adoption d'un mode descriptif tri-dimensionnel des "outils" lexicaux et grammaticaux nous semble tout aussi justifiée. Ces principes descriptifs étant posés, il reste évidemment à formuler le détail du parcours grammatical.

Sur le plan de la modélisation linguistique, après avoir été longtemps à l'étroit dans un paradigme immanentiste et phrastique, on risque subitement d'avoir l'impression de disposer d'un cadre de travail élargi au point de poser des problèmes de "remplissage" descriptif pour les nouvelles dimensions postulées au sein des signifiés. Ces questions se posent notamment pour les termes les plus faciles à gérer dans le cadre saussurien. Il semble difficile par exemple de définir un caractère énonciatif ou textuel particulier au mot escalier. Probablement parce que ce terme est recevable quels que soient la situation d'énonciation ou le texte envisagés. Mais le bénéfice intégratif de cette représentation sémantique paraît tout de même suffisant pour que l'on explore un espace descriptif cognitivement fondé, à la fois dans ses possibilités d'élargissement, mais aussi dans les interrogations que posent ces dernières.

Notre dernier mot ne peut aller que dans le sens d'un plaidoyer pour une meilleure interaction entre ces trois disciplines que sont la psychologie cognitive, la linguistique et la didactique (Barbazan, à paraître d). La première propose des repères qui doivent être impérativement pris en compte par les linguistes soucieux de descriptions cognitivement recevables et a fortiori par les didacticiens. On peut espérer que ce fond cognitif commun justifie alors une réelle interaction entre une linguistique non colonialiste et une didactique confiante en son intégrité.

\section{Références bibliographiques}

Arditty J. / Vasseur M.-T. (1999). Interaction et langue étrangère. Langages $n^{\circ} 134$.

Adam J.-M. (1992). Les textes : types et prototypes. Paris : Nathan.

Adam J.-M. (1999). Linguistique textuelle. Des genres de discours aux textes. Paris : Nathan.

Barbazan M. (2002). Le discours indirect libre : éléments cognitifs de décodage et implications dialogiques pour le signifié de l'imparfait. Nouveaux Cahiers d'Allemand 20 / 1, 65-91.

Barbazan M. (2004). L'interaction entre modélisation linguistique et modélisation psycholinguistique: l'apprentissage des temps verbaux du français par des apprenants germanophones. Thèse de Doctorat. Toulouse, Université de Toulouse-Le Mirail.

Barbazan M. (2006). Le temps verbal. Dimensions linguistiques et psycholinguistiques. Toulouse, Presses Universitaires du Mirail.

Barbazan M. (2007a). Les temps verbaux du passé : quelle didactique pour le FLE ? Repères linguistiques et cognitifs. Actes du colloque Didcog 2, 19-21 septembre 2007, Université de Toulouse-Le Mirail.

Barbazan M. (2007b). Le trait [+/- allocutif] : un principe explicatif de l'opposition du passé simple et du passé composé. Romanische Forschungen 2007 / 4, n¹19, 429-463. 
Barbazan M. (à paraître a). De la psycholinguistique à la stylistique : discours indirect libre et polyphonie en contexte. A paraître dans l'Information Grammaticale, octobre 2008.

Barbazan M. (à paraître b). Héritage / acquis grammatical, métalangage, activités grammaticales : quelles options didactiques pour une description du système verbal en FLE ? Colloque International Enseigner les structures langagières en FLE. Bruxelles, 20-22 mars 2008, Université Libre de Bruxelles.

Barbazan M. (à paraître c). De la grammaire explicite à l'intériorisation : une question d'adéquation linguistique, didactique et cognitive. Article en cours d'examen par la revue PArole.

Barbazan M. (à paraître d). Modèles explicatifs, modèles prédictifs : pour une interaction effective entre linguistique et cognition. Article en cours d'examen par la revue Cahiers Chronos.

Benveniste E. (1966). Problèmes de linguistique générale. Tome I. Paris : Gallimard.

Benveniste E. (1974). Problèmes de linguistique générale. Tome II. Paris : Gallimard.

Berrendonner A. (1981). Éléments de pragmatique linguistique. Paris : Éditions de Minuit, coll. Propositions.

Besse H. / Porquier R. (1984). Grammaires et didactique des langues. Paris : Hatier.

Bruner J. S. (1983) Le développement de l'enfant : savoir faire, savoir dire. Paris : PUF.

Confais J.-P. (2001), Modèles textuels et cognition. Aspects psycholinguistiques de la compréhension des textes. Garcia-Debanc C. et al. (2001). 155-174.

Charolles M. / Combettes B. (2001) de la phrase au discours: rupture et continuité. Garcia-Debanc C. et al. (2001).119-142.

Cuq J.-P. (1996). Une introduction à la didactique de la grammaire en français langue étrangère. Paris : Didier.

Denhière G. (1984). Il était une fois... Lille : Presses Universitaires de Lille.

Dubois D. et al. (1993). Sémantique et cognition. Catégories, prototypes, typicalité. Paris : CNRS Editions.

Ducrot O. (1991). Dire et ne pas dire. Paris : Hermann.

Fayol M. (1994 / 1985). Le récit et sa construction. Une approche de la psychologie cognitive. Lausanne : Delachaux et Niestlé.

Fayol M. (1997). Des idées au texte. Psychologie cognitive de la production verbale, orale et écrite. Paris : PUF.

Fayol M. (2001). Produire des textes à l'écrit. Un état des recherches psycholinguistiques. Garcia-Debanc C. et al. (2001). 305-318.

Fayol M. et al. (1992). Psychologie cognitive de la lecture. Paris : PUF.

Fuchs C. / Le Goffic P. (1992). Les linguistiques contemporaines. Repères théoriques. Paris : Hachette.

Garcia-Debanc C./Confais J.-P./Grandty M. (2001). Quelles grammaires enseigner à l'école et au collège ? Discours, genre, texte, phrase. Toulouse : Delagrave/CRDP Midi-Pyrénées.

Gaonac'h D. (1991). Théories d'apprentissage et acquisition d'une langue étrangère. Paris : Didier

Gaonac'h D. / Larigauderie P. (2000). Mémoire et fonctionnement cognitif. La mémoire de travail. Paris : Colin.

Germain C. / Séguin H. (1995). Le point sur la grammaire. Paris : CLE International.

Jacques F. (1985). Dialogiques II. L'espace logique de l'interlocution. Paris : PUF.

Kerbrat-Orecchioni C. (1997 / 1980). L'énonciation. De la subjectivité dans le langage. Paris : Colin.

Kleiber G. (1997). Sens, référence et existence : que faire de l'extra-linguistique ? Siblot P. (éd.) Langue, praxis et production de sens. Langages 127, 9-37.

Le Ny J.-F. (1979). La sémantique psychologique. Paris : PUF.

Maingueneau D. (1993). Eléments de linguistique pour le texte littéraire. Paris : Dunod.

Maingueneau D. (2001). Le recours à la notion de discours en didactique. Quelques réflexions. Garcia-Debanc C. et al. (2001). 15-22.

Mondada L. / Pekarek Doehler S. (2000). Interaction sociale et cognition située : quels modèles pour la recherche sur l'acquisition des langues? Acquisition et Interaction en Langue Étrangère $n^{\circ} 12$ (en ligne)

Puren C. (1988). Histoire des méthodologies de l'enseignement des langues. Paris : Nathan.

Rosier L. (1999). Le discours rapporté. Histoire, théories, pratiques. Paris / Bruxelles : Duculot.

Véronique D. / Vion R. (eds.) (1995). Modèles de l'interaction verbale. Publications de l'Université de Provence.

Vigner G. (2004). La grammaire en FLE. Paris : Hachette.

Vuillaume M. (2000). La signalisation du style indirect libre. Mellet S. / Vuillaume M. (éd.) (2000), Le style indirect libre et ses contextes. Cahiers Chronos 5. Amsterdam, Rodopi, 107-130.

Vygotski L. (1997 / 1934). Pensée et langage. Paris : La dispute.

Weinrich H. (1973). Le temps. Paris : Seuil, coll. Poétique.

Weinrich H. (1982). Textgrammatik der französischen Sprache. Stuttgart: Klett. 